\title{
AVALIAÇÃO DA EFICIÊNCIA DE DELTAMETRINA (K-OTHRINE CE) NO CONTROLE DE LUTZOMYIA LONGIPALPIS (DIPTERA: PSYCHODIDAE), NO MUNICÍPIO DE SANTA RITA, PARAÍBA, BRASIL
}

\author{
Carlos Brisola Marcondes e João Agnaldo Nascimento
}

\begin{abstract}
Deltametrina em concentrado emulsionável foi aplicada em doses de 12,5, 25,0 e $50,0 \mathrm{mg} / \mathrm{m}^{2}$, em domicílios de área próxima do litoral da Paraiba, onde eram relatados casos esporádicos de leishmaniose visceral. Houve uma redução marcante nas populaçzes de vários artrópodes, incluindo Lutzomyia longipalpis. Este inseto, no entanto, foi encontrado novamente em contato com paredes tratadas, 14 e 21 dias após a pulverização. A análise estatística indicou controle significativo em apenas dois periodos de quatro semanas, com extrema irregularidade nas quantidades capturadas.
\end{abstract}

Palavras-chaves: Deltametrina. Phlebotominae. Lutzomyia. longipalpis. Controle. Leishmaniose visceral.

Ocontrole de flebotomíneos é muito importante para a profilaxia da leishmaniose visceral ${ }^{2} \mathrm{e}$ foram obtidos bons resultados com o uso de DDT ${ }^{2}$. No entanto, foi constatada resistência de flebotomíneos a organoclorados na India ${ }^{13}$.

Devido aos bons resultados obtidos com o uso de deltametrina, no controle de vários insetos ${ }^{3}$, realizamos avaliação deste piretróide em Lutzomyia longipalpis, emárea no leste do Estado da Paraŕba.

\section{MATERIAL E MÉTODOS}

O teste foi realizado no Município de Santa Rita (708'S e 34\%59'W), a 20 metros acima do nível do mar e a cerca de $20 \mathrm{~km}$ do litoral da Paraíba. A pluviosidade anual média é de $1415,8 \mathrm{~mm}$, com $50,01 \%$ concentrados em abrilmaio-junho ${ }^{10}$. A área estudada, de cerca de $3 \mathrm{~km}^{2}$, abrange, segundo mapa da Fundação Nacional da Saúde(FNS, ex-SUCAM), as localidades de Bairro Sol Nascente e Planalto de Santa Rita, fica ao leste e é contígua ao Conjunto Habitacional Tibiri II. A vegetação é arbustiva e rasteira ("capoeira"), com algumas pequenas plantações peridomiciliares de

\footnotetext{
Núcleo de Medicina Tropical da Universidade Federal da Paraiba, João Pessoa, PB, apresentado no XI Congresso da Sociedade Brasileira de Parasitologia, Fortaleza, 1985.

Endereço para correspondência: Prof. Carlos Brisola Marcondes. Depto. de Microbiologia e Parasitologia/CCB/ UFSC, Campos Trindade, 88040-900 Florianópolis, SC. Recebido para publicação em 25/09/92.
}

feijão, mandioca e batata-doce. $O$ solo é arenoso e areno-argiloso.

$O$ inseticida foi aplicado em concentrado emulsionável (CE), em junho de 1984, em 58 domicílios, sendo $16 \mathrm{com} 50 \mathrm{mg} / \mathrm{m}^{2}$ (grupo A), 15 com $25 \mathrm{mg} / \mathrm{m}^{2}$ (B), $13 \mathrm{com} 12,5 \mathrm{mg} / \mathrm{m}^{2}$ (C) e $14 \mathrm{com}$ querosene em água a 1:100 (D-controle), com distribuição aleatória nos grupos. Foram usadas bombas Hudson-Perty da SUCAM, conforme metodologia descrita anteriormente ${ }^{9}$, aplicando-se no intradomićlio e nas poucas instalações peridomiciliares existentes.

A partir da semana seguinte à da aplicação, as casas foram separadas em dois grupos de $29 \mathrm{e}$, em cada sábado, das 18 às 21 horas, quatro funcionários da FNS examinaram as componentes de um grupo, por um período total de 36 semanas. Foram capturados flebotomíneos nas paredes internas, com capturadores manuais de sucção. Os insetos foram preservados em álcool $70^{\circ} \mathrm{GL}$ e, após clarificação, foram montados em lactofenol e identificados.

A comparação dos quatro grupos foi feita por análise não-paramétrica de Kruskal-Willis e a do grupo tratado com o controle com teste " $t$ " de Student.

\section{RESULTADOS}

Os efeitos imediatos da pulverização foram muito marcantes, com grande mortalidade de vários artrópodes nocivos (aranhas, baratas, quilópodos, etc) e desaparecimento de flebotomíneos eculicídeos, 
Marcondes $C B$, Nascimento JA. Avaliação da eficiência de deltrametrina ( $K$-Othrine $C E$ ) no controle de Lutzomyia longipalpis (Diptera: Psychodidae), no município de Santa Rita, Paraiba, Brasil. Revista da Sociedade Brasileira de Medicina Tropical 26:15-18, jan-mar, 1993.

sem efeitos tóxicos evidentes em pessoas e animais domésticos.

L. longipalpis foi predominante nas amostras, com pequenas quantidades de L. evandroi. Só foram usadas para cálculos as amostras intradomiciliares da primeira, obtidas em domicílios sem interferência humana evidente, como pintura de parede posterior à aplicação, fumaça ou uso de outros inseticidas.

A Tabela 1 mostra as quantidades de $L$. longipalpis capturadas e usadas para cálculos, nos quatro grupos de casas, em cada período de quatro semanas. A Tabela 2 mostra as médias geométricas modificadas $(\mathrm{Mw})^{1}$, nos quatro grupos e a Tabela 3 mostra as $\mathrm{Mw}$ do grupo tratado e do controle.

Tabela 1 - Quantidade de L. longipalpis capturadas em casas dos grupos A (deltametrina a $50 \mathrm{mg} / \mathrm{m}^{2}$ ), $B\left(25 \mathrm{mg} / \mathrm{m}^{2}\right), C\left(12,5 \mathrm{mg} / \mathrm{m}^{2}\right)$ e D (controle) nos nove periodos de quatro semanas, em Santa Rita, Paraiba.

\begin{tabular}{|c|c|c|c|c|c|c|c|c|c|c|}
\hline \multirow[b]{2}{*}{ Grupos } & \multicolumn{10}{|c|}{ Períodos } \\
\hline & $1^{\circ}$ & $2^{0}$ & $3^{\circ}$ & $4^{\circ}$ & $5^{\circ}$ & $6^{\circ}$ & $7^{\circ}$ & $8^{\circ}$ & $9^{\circ}$ & Total \\
\hline $\bar{A}$ & 6 & 21 & 20 & 33 & 94 & 62 & 24 & 43 & 32 & 335 \\
\hline B & 21 & 9 & 21 & 2 & 4 & 8 & $\cdot 1$ & 3 & - & 69 \\
\hline C & 10 & 3 & 1 & 34 & 4 & 28 & 6 & 1 & 27 & 114 \\
\hline D & 48 & 62 & 40 & 35 & 70 & 19 & 15 & 44 & 5 & 338 \\
\hline Total & 85 & 95 & 82 & 104 & 172 & 117 & 46 & 91 & 64 & 856 \\
\hline
\end{tabular}

Tabela 2 - Quantidades médias $(\mathrm{Mw})$ de $\mathrm{L}$. longipalpis capturadas em casas dos grupos A (deltametrina a $\left.50 \mathrm{mg} / \mathrm{m}^{2}\right)$, B $\left(25 \mathrm{mg} / \mathrm{m}^{2}\right), C\left(12,5 \mathrm{mg} / \mathrm{m}^{2}\right)$ nos nove períodos de quatro semanas, em Santa Rita, Paraiba.

\begin{tabular}{|c|c|c|c|c|c|c|c|c|c|}
\hline \multirow[b]{2}{*}{ Grupos } & \multicolumn{9}{|c|}{ Períodos } \\
\hline & $1^{0 *}$ & $2^{0 *}$ & $3^{\circ}$ & $4^{\circ}$ & $5^{\circ}$ & $6^{\circ}$ & $7^{\circ}$ & $8^{\circ}$ & $9^{\circ}$ \\
\hline A & 0,1134 & 0,3005 & 0,2497 & 0,4122 & 0,9820 & 0,8147 & 0,2084 & 0,7608 & 0,5740 \\
\hline B & 0,4025 & 0,2859 & 0,5474 & 0,0904 & 0,1487 & 0,2697 & 0,0651 & 0,1962 & 0,0000 \\
\hline C & 0,1513 & 0,0937 & 0,0416 & 0,3555 & 0,0993 & 0,5675 & 0,2134 & 0,0651 & 0,7061 \\
\hline D & 0,8348 & 0,9957 & 0,4948 & 0,5484 & 0,7905 & 0,3897 & 0,2561 & 0,8570 & 0,1365 \\
\hline
\end{tabular}

* diferenças significativas entre grupos.

Tabela 3 - Quantidades médias $(M w)$ de $\mathrm{L}$. longipalpis em casas do grupo tratado com deltametrina e controle nos nove periodos de quatro semanas, em Santa Rita, Paraiba.

\begin{tabular}{lccccccccc}
\hline & \multicolumn{10}{c}{ Períodos } \\
\cline { 2 - 9 } Grupos & $1^{0 *}$ & $2^{0 *}$ & $3^{0}$ & $4^{0}$ & $5^{0}$ & $6^{0}$ & $7^{0}$ & $8^{\circ}$ & $9^{0}$ \\
\hline Tratado & 0,2076 & 0,2135 & 0,2768 & 0,2993 & 0,4321 & 0,5550 & 0,1707 & 0,3127 & 0,4899 \\
Controlado & 0,8348 & 0,9957 & 0,4948 & 0,5484 & 0,7905 & 0,3897 & 0,2561 & 0,8570 & 0,1365 \\
\hline
\end{tabular}

* diferenças significativas entre grupos. 
Marcondes CB, Nascimento JA. Avaliação da eficiência de deltrametrina ( $K$-Othrine $C E$ ) no controle de Lutzomyia longipalpis (Diptera: Psychodidae), no municipio de Santa Rita, Paraiba, Brasil. Revista da Sociedade Brasileira de Medicina Tropical 26:15-18, jan-mar, 1993.

Apesar do uso de $\mathrm{Mw}$, algumas médias foram muito aumentadas pela contribuição de certas amostras, como em A no $5^{\circ}$ e no $6^{\circ}$. ciclos. Em ambas as comparaçôes, só foram evidenciadas diferenças significativas entre os grupos nos dois primeiros períodos de quatro semanas.

Foram obtidos flebotomíneos numa casa do grupo A e numa do grupo $B$, duas semanas após a pulverização, e numa do grupo $B$ e numa de $C$, três semanas após aquela. Em várias das casas, não obtivemos insetos em todo o período.

\section{DISCUSSÃO}

Apesar da extrema irregularidade das amostras, o fato de ter sido encontrado $L$. longipalpis em contato com paredes tratadas há duas ou três semanas indica poderem eles suportar pelo menos um curto contato com inseticida. Como num teste no Espírito Santo $^{4}$, não foi feita uma avaliação prévia da variação estacional nas populações de L. longipalpis, mas o fato do estudo de Santa Rita ter grupo controle, sem deltametrina, nas mesmas localidades, permitiu a realização de análise estatística adequada.

$O$ método de amostragem ideal para a avaliação dos efeitos do inseticida seria a comparação do numero de picadas infectantes do flebotomíneo em iscas humanas no intradomicnlio, de realização muito difícil. Assim, têm sido usados outros métodos, como as armadilhas luminosas ${ }^{4} 8$ e a captura em paredes. Certos flebotomíneos podem sobreviver ao contato forçado de 15 minutos com jaquetas impregnadas com permetrina, morrendo até 30 minutos após ${ }^{15}$. É possível que o fato de os insetos do presente estudo terem sido postos no álcool logo após a captura tenha mascarado a mortalidade tardia, após o contato com superfícies tratadas com deltametrina. Esta demora para matar flebotomíneos contrasta com estudos similares em outros insetos ${ }^{16} 17$.

Os resultados deste trabalho indicam um efeito significativo de curta duração sobre L. longipalpis. Estes resultados contrastam com os de teste na mesma espécie na Bolívia ${ }^{8}$. A formulação em concentrado emulsionável, usada em Santa Rita pode ser menos eficiente em superfícies absorventes que a de pó molhável usada na Bolívia, como já observado para triatomíneos ${ }^{11}$. L. longipalpis é considerada atualmente um complexo de espécies ${ }^{18}$.
É possivel que ocorram variações regionais no comportamento e na susceptibilidade dos componentes do complexo ao inseticida. Os resultados de testes no Estado do Espírito Santo e na Argentina ${ }^{414}$, com L. intermedia, também indicam maior ação residual do inseticida, possivelmente ligadas a diferenças de formulação deste ou de susceptibilidade das espécies.

Testes realizados em região semi-árida do Ceará, na Grécia, na Itália, na Palestina e na Índia 25612 , o último com Malation e os outros com DDT, evidenciaram redução marcante e prolongada dos flebotomíneos em domicílios. No entanto, o surgimento de resistência de flebotomíneos a DDT na Índia ${ }^{13}$ torna conveniente a realização de estudos mais detalhados sobre o efeito de deltametrina em flebotomíneos de importância médica. Foi relatada a ocorrência de resistência cruzada entre organoclorados e piretróides ${ }^{3}$.

Apesar de referências da população local do aumento de ataque por flebotomíneos no início do ano, não conseguimos detectar variações estacionais, no período estudado. O conhecimento destas variações na área de Santa Rita é importante para compreensão de sua biologia e papel como transmissor e otimização do controle.

\section{AGRADECIMENTOS}

Ao Dr. Cláudio Teixeira Pinto, da Quimio Prod. Quim. Com. Ind. S.A., pelo fornecimento do inseticida e apoio ao desenvolvimento do trabalho, e ao Dr. João Pequeno Madruga, na época Diretor Regional da FNS, pela colaboração.

\section{SUMMARY}

Deltamethrin in emulsion concentrate was applied in doses of $12.5,25.0$ and $50.0 \mathrm{mg} / \mathrm{m}^{2}$, in households near the littoral of the State of Paraiba, where sporadic cases of visceral leishmaniasis were reported. There was a remarkable decrease in populations of several arthropods, including Lutzomyia longipalpis. This insect, however, was found again in some treated walls, 14 and 21 days after spraying. Statistical analysis indicated significant control for only two four-weeks periods, with extreme irregularity in the quantity captured.

Key-words: Deltamethrin. Phlebotominae. Lutzomyia longipalpis. Control. Visceral leishmaniasis. 
Marcondes $C B$, Nascimento JA. Avaliação da eficiência de deltrametrina (K-Othrine $C E)$ no controle de Lutzomyia longipalpis (Diptera: Psychodidae), no município de Santa Rita, Paraíba, Brasil. Revista da Sociedade Brasileira de Medicina Tropical 26:15-18, jan-mar, 1993.

\section{REFERÊNCIAS BIBLIOGRÁFICAS}

1. Bidlingmayer WL. The use of logarithms in analysis trap collections. Mosquito News 29:635-640, 1969.

2. Deane LM, Deane MP, Alencar JE. Observações sobre o combate ao Phlebotomus longipalpis pela dedetização domiciliária, em área endêmica de calazar no Ceará. Revista Brasileira de Malariologia e Doenças Tropicais 7:131-140, 1955.

3. Elliot $M$, Janes NF, Potter C. The future of pyrethroids in insect control. Annual Review of Entomology 23:443-469, 1978.

4. Falcão AL, Falcão AR, Pinto CT, Gontijo CMF, Falqueto A. Effect of deltamethrin spraying on the sandfly populations in a focus of American cutaneous leishmaniasis. Memórias do Instituto Oswaldo Cruz 86:399-404, 1991.

5. Hertig M. Phebotomus and residual DDT in Greece and Italy. American Journal of Tropical Medicine 29:773-809, 1949.

6. Jacusiel F. Sandfly control with DDT residual spray. Field experiment in Palestine. Bulletin of Entomological Research 38:479-488, 1947.

7. Joshi GC, Kaul SM, Wattal BL. Susceptibility of sandflies to organochlorine insecticides in Bihar (India) - further reports. Journal of Communicable Diseases 11:209-213, 1979.

8. Le Pont F, Mariscal Padilla J, Desjeux P, Richard A, Mouchet J. Impact de pulverisations de deltamethrine dans un foyer de leishmaniose de Bolivie. Annales de la Societé Bèlge de Mèdecine Tropicale 69:223-232, 1989.

9. Marcondes CB, Pinto CT. Avaliação da eficiência de deltametrina (K-Othrine 50FW), em doses baixas, no controle de triatomíneos em São Sebastião do Umbuzeiro (Paraíba). Revista da Sociedade Brasileira de Medicina Tropical 22:85-90, 1989.

10. Nimer E. Pluviometria e recursos hídricos de Pernambuco e Paraíba. Instituto Brasileiro de Geografia e Estatística, Rio de Janeiro, 1979.
11. Oliveira Filho AM. Field trials of 3 formulations of deltamethrin in houses colonized by Triatoma infestans. In: Resumos da $11^{\text {a }}$ Reunião de Pesquisa Básica em Doença de Chagas, Caxambu p.170, 1984.

12. Pandya AP. Impact of antimalaria house spraying on phlebotomid population in Surat District, Gujarat. Indian Journal of Medical Research 78:354-360, 1983.

13. Pandya AP, Mehta NR, Niyogi AK. The development of resistance in sandfly to hydrocarbon insecticides in Gujarat State. Journal of Indian Association of Previdence and Social Medicine, annual number p.111-114, 1975.

14. Ripoll C, Remondegui C, Romano F, Caorlin O, Rovetti E. Brote de leishmaniasis tegumentária en la provincia de Jujuy, Argentina. In: Resumos del II Congreso Argentino de Protozoologia, La Falda, Córdoba, Argentina p.51, 1987.

15. Schreck CE, Kline DL, Chaniotis $B N$, Wilkinson $N$, McGovern TP, Weidhaas DE. Evaluation of personal protection methods against phlebotomine sandflies including vectors of leishmaniasis in Panama. The American Journal of Tropical Medicine and Hygiene 31:1046-1053, 1982.

16. Schreck CE, Posey K, Smith D. Durability of permethrin as a potential clothing treatment to protect against blood-feeding arthropods. Journal of Economic Entomology 71:397-400, 1978.

17. Schreck CE, Snoody EL, Mount GA. Permethrin and repellents as clothing impregnants for protection from the lone star tick. Journal of Economic Entomology 73:436-439, 1980.

18. Ward RD, Phillips A, Burnet B, Marcondes CB. The Lutzomyia longipalpis complex: reproduction and distribution. In: Service MW (ed) Byosystematics of haematophagous insects. Claredon Press, Oxford p.257-269, 1988. 